\title{
Duplication and transcriptional divergence of three Kunitz protease inhibitor genes that modulate insect and pathogen defenses in tea plant (Camellia sinensis)
}

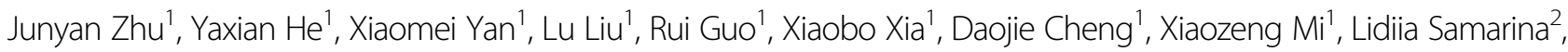
Shenrui Liu', Enhua Xia ${ }^{1}$ and Chaoling Wei ${ }^{1}$

\begin{abstract}
Kunitz protease inhibitors (KPIs) are ubiquitous in plants and act as crucial compounds in defense responses against insect attack and pathogen infection. However, the influence of gene duplication on the postdivergence of the CsKPI genes involved in biotic stresses in tea plant is not well known. Here, we identified three CSKPI genes from tea plant (Camellia sinensis) and characterized their expression and evolutionary patterns among plant species. We found that CSKPI1, CSKPI2, and CSKPI3 diverged from their common ancestor 72.94 million years ago (MYA), and the tandem duplication of CSKPI2 and CsKPI3 occurred 26.78 MYA. An in vitro protein assay showed that the three CsKPI proteins were functional and inhibited the production of $p$-nitroanilide (PNA) from an artificial substrate. The three CsKPI-GFP fusion proteins localized to the cytoplasm. We showed that salicylic acid (SA) and transcripts of CsKPI2 and CsKPI3 significantly accumulated after infection with Glomerella cingulata. The application of exogenous SA stimulated the high expression of both CSKPI2 and CSKPI3 by activating cis-elements within their promoters. Under Ectropis oblique attack, CSKPII expression and jasmonic acid (JA) levels were more abundant in both insect-damaged leaf tissues and undamaged neighboring leaves. The application of jasmonic acid methyl ester elicited high expression levels of CsKPI1, suggesting that CSKPI1 accumulation requires JA production in tea plant. The overall findings suggest that the transcriptional divergence of KPI genes after duplication led to the specialized role of CsKPI1 in the physiological response to insect stress; the functional conservation between CSKPI2 and CSKPI3 confers resistance to pathogen infection in tea plant.
\end{abstract}

\section{Introduction}

The tea plant (Camellia sinensis) is an important economic woody crop that is cultivated worldwide ${ }^{1}$. Tea leaves are used to make tea beverages, which have a rich taste and confer health benefits ${ }^{2}$. The tea processing

\footnotetext{
Correspondence: Chaoling Wei (weichl@ahau.edu.cn)

${ }^{1}$ State Key Laboratory of Tea Plant Biology and Utilization/Key Laboratory of Tea Biology and Processing, Ministry of Agriculture, Anhui Agricultural University, West 130 Changjiang Road, Hefei 230036 Anhui, People's Republic of China

${ }^{2}$ Russian Research Institute of Floriculture and Subtropical Crops, 354002Yana Fabritsiusa st. 2/28, Sochi, Russian Federation

These authors contributed equally: Junyan Zhu, Yaxian He, Xiaomei Yan
}

method, genetic variation in tea cultivars and environmental stresses are crucial factors that affect the quality of tea leaves ${ }^{3}$. To date, it is widely known that many abiotic and biotic stresses, such as drought ${ }^{4}$, low temperature ${ }^{5}$, insect attack ${ }^{6}$, and pathogen infection ${ }^{7}$, can induce a series of defense responses in tea plant.

Protease inhibitors (PIs) are present in almost all organisms, including animals, plants, and microorganisms; they play key regulatory roles in a wide range of biological processes ${ }^{8,9}$. They function by directly or indirectly blocking active centers of target proteases to strictly control their activities ${ }^{8,10}$. In vitro, $\mathrm{N}-\alpha$-benzoyl-DL-arginine-4- 
nitroanilide (BAPNA) can act as a trypsin substrate to catalyze the production of $p$-nitroanilide (PNA); PI proteins suppress the activity of trypsin and reduce the amount of PNA production. PIs are grouped into families and are induced in plants in response to injury or attack by insects or pathogens ${ }^{11,12}$. In Arabidopsis, serpin 1 proteins function as potent inhibitors that destabilize metacaspase-like proteins in vivo and function in the plant immune response ${ }^{13}$. Kunitz PIs (KPIs) are members of a subfamily of PIs involved in biotic defense; they function by repressing the digestive processes of insects and reducing fungal lesion development ${ }^{9,14}$. In Pithecellobium dumosum, PdPI3.1 and PdPI3.2 are members of the KPI family and affect the digestive enzymes of insect larvae of diverse orders ${ }^{15}$. A serine PI (Kunitz-type inhibitor designated PKI1) isolated from potato showed direct inhibitory activity on Botrytis cinerea strains as reported by Hermosa et al. ${ }^{16}$.

Ohno proposed a theory that gene duplication (GD) events represent the primary source of genetic novelty leading to speciation ${ }^{17-20}$. Subsequently, the subfunctionalization (SF) model was proposed: the shared functions between duplicated genes diverge over time, and these genes evolve into functionally distinct proteins, with each daughter gene specializing in a subset of functions of the ancestral gene ${ }^{21,22}$. Force et al. (1999) then proposed the duplication-degeneration-complementation (DDC) model; degenerative mutations accumulate within duplicated genes for a period of time, which then undergo functional specialization by complementary partition of ancestral functions ${ }^{21,22}$. The evolution of a gene family often results from polyploidization, whole-genome duplication (WGD), or tandem (linked) or segmental (unlinked) duplications; these have been frequent in the evolutionary history of flowering plants and have shaped the evolutionary trajectory of genomes and genes ${ }^{3,23-25}$. Tandem duplication can generate a copy of several genes within the same scaffold or chromosome; these events often include genes from the same networks or pathways and can accelerate the divergence of gene function. With the recent release of whole genome sequences, multiple gene families were found to undergo tandem GD in addition to functional differentiation; examples, include gene families such as dof, expansin, and phenylalanine ammonialyase ${ }^{20,26,27}$. However, there is limited knowledge regarding the mechanism of duplication events in the KPI gene family and their evolutionary trajectories in tea plant.

Plant hormones are complex and important signaling molecules that modulate many aspects of plant development and defense ${ }^{28}$. Salicylic acid (SA) is a phenolic compound that functions as a key signal in regulating disease resistance. It acts through effector-triggered immunity (ETI), thereby modulating the activities of diverse groups of defense-related genes; examples include those genes encoding PI proteins and several families of pathogenesis-related (PR) proteins ${ }^{8,29}$. Jasmonic acid (JA) is a well-known hormone regulator that mediates defense responses to insect attack $^{30,31}$. Previous studies have indicated that PIs and JA are both strongly induced in leaves damaged by insect feeding, which implies that JA may induce the expression of PI genes and synergistically defend against insect attack ${ }^{6,12,32}$. In Nicotiana attenuata, JA and trypsin proteinase inhibitors are regulated by the BAK1 gene in response to herbivory ${ }^{33}$. Previous studies also indicate that plants can perceive events locally and transduce a defense signal to mount a broad and systemwide response to stresses (i.e., systemic acquired resistance (SAR) $)^{34,35}$. Although the SAR model proposes that SA and MeSA initiate SAR in plants, recent studies demonstrate that JA is also a crucial signal molecule in the SAR response; JA accumulates via de novo JA biosynthesis and then acts to transmit the signal and activate the expression of downstream-related defense genes ${ }^{34,36}$. Truman et al. ${ }^{37}$ proposed that genes involved in jasmonate biosynthesis are upregulated within $4 \mathrm{~h}$, and JA then diffuses to undamaged neighboring leaves. SAR can be activated by foliar JA application and is abolished in mutants impaired in JA synthesis or the JA response ${ }^{37}$. Despite these studies exploring JA-mediated regulation of the SAR response, very little is known about whether KPIs are involved in SAR and which potential signals regulate KPIs under the SAR response.

Our study aims to determine the function of three CsKPI genes in tea plant. We initially identified these three CsKPIs from the tea plant genome and explored their evolutionary relationships with various plant species. Heterologous expression and subcellular localization were employed to characterize the functions of these CsKPIs. We further examined the expression patterns of the three CsKPIs in response to pathogen infection and insect attack. CsKPI2 and CsKPI3 exhibited high mRNA accumulation in response to pathogen infection, and this response may require SA induction. We observed that CsKPI1 may be involved in SAR based on its expression, which was strongly induced both by localized insect feeding and in undamaged neighboring leaves. Promoter activity analysis suggested that CsKPI1 is activated by JA, whereas CsKPI2 and CsKPI3 are activated by SA. Our results improve the understanding of the diversified functions of CsKPIs resulting from duplication in tea plant and highlight the underlying roles of CsKPIs in the SAR response.

\section{Results \\ Identification of tea CsKPIs and evolution analysis in plant species}

To identify tea CsKPI genes, KPI amino acid reference sequences from four closely related species were used to 
search the tea genome (C. sinensis) $)^{3}$. Three CsKPIs were identified and then validated by both reverse transcription polymersae chain reaction (RT-PCR) and Sanger sequencing. The full-length sequence of CsKPI2 was obtained by rapid amplification of cDNA ends (RACE). To explore important structural regions of CsKPI proteins, we aligned three CsKPIs with seven KPIs from Theobroma cacao (2), Vitis vinifera (3), and Populus trichocarpa (2) plant species. The alignment showed that two residues [arginine (Arg, R) and lysine (Lys, K)] were highly conserved across species (Fig. 1). We further observed a high amino acid sequence similarity (75\%) between CsKPI2 and CsKPI3, but CsKPI1 was less similar to CsKPI2 (53\%) and CsKPI3 (51\%); this suggested that CsKPI1 may have functionally diverged from CsKPI2 and CsKPI3.

To investigate the evolutionary relationship of CsKPIs among plant species, we constructed a phylogenetic tree using a total of $19 \mathrm{KPI}$ gene sequences from ten plant species (Fig. 2). The 19 KPIs were divided into two clusters based on their evolutionary relationship and sequence similarities, and the three CsKPIs were grouped into the eudicot category. Moreover, CsKPI2 and CsKPI3 were tandemly located within the same scaffold (Supplementary Table. S1). This suggested that a tandem duplication event occurred between these two genes 26.78 MYA, but the separation between these two genes and CsKPI1 occurred 72.94 MYA (Supplementary Fig. S1). A similar duplication event was observed in some eudicots, such as $P$. trichocarpa (Potri.017G153400.1 and Potri.017G153300.1), V. vinifera (GSVIVT01012922001 and GSVIVT01012936001), T. cacao (Thecc1EG025233t1 and Thecc1EG025234t1), and Aquilegia coerulea (Aqcoe6G187000.1 and Aqcoe6G187100.1). Notably, no KPIs were identified in lower plants, such as Dunaliella salina, Physcomitrella patens, and Selaginella moellendorffi, until the occurrence of grass plants, in which a single KPI gene was first identified in Oryza sativa.

\section{Activity and subcellular localization of CsKPI1, CsKPI2, and CsKPI3}

As indicated by the close but distinct phylogenetic relationship between CsKPI1, CsKPI2, and CsKPI3, these genes may exhibit unique enzymatic properties or differ in their biological functions. Heterologous expression and enzymatic measurements were necessary to determine whether the CsKPI1, CsKPI2, and CsKPI3 proteins were functional trypsin inhibitors. We cloned the CDS regions of the three CsKPIs into pGEX-4T-1 and obtained the recombinant proteins by heterologous expression of each gene in E. coli. Recombinant proteins were purified to improve the efficiency of the enzymatic reactions and to eliminate any interference from nontarget proteins.

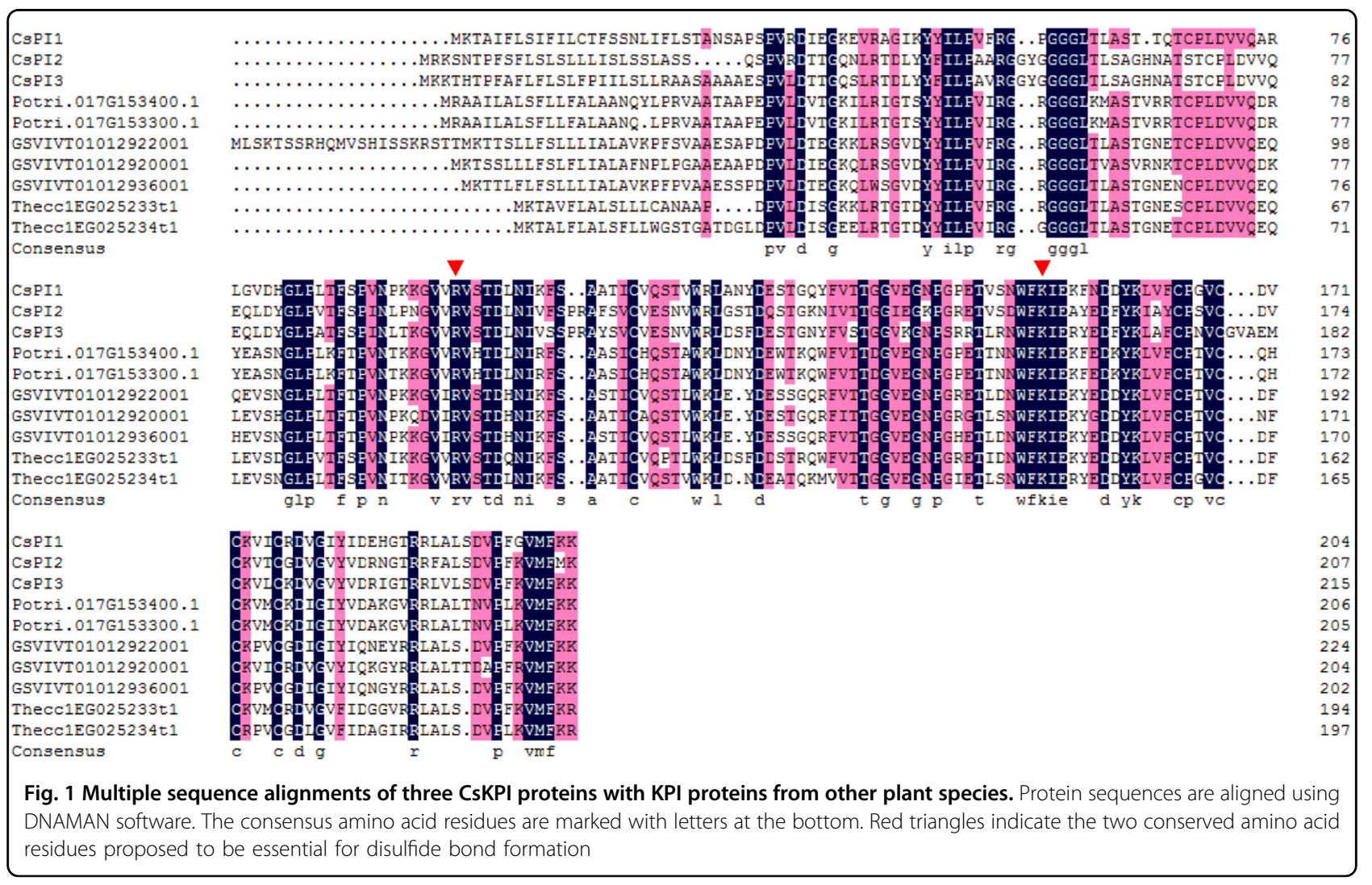




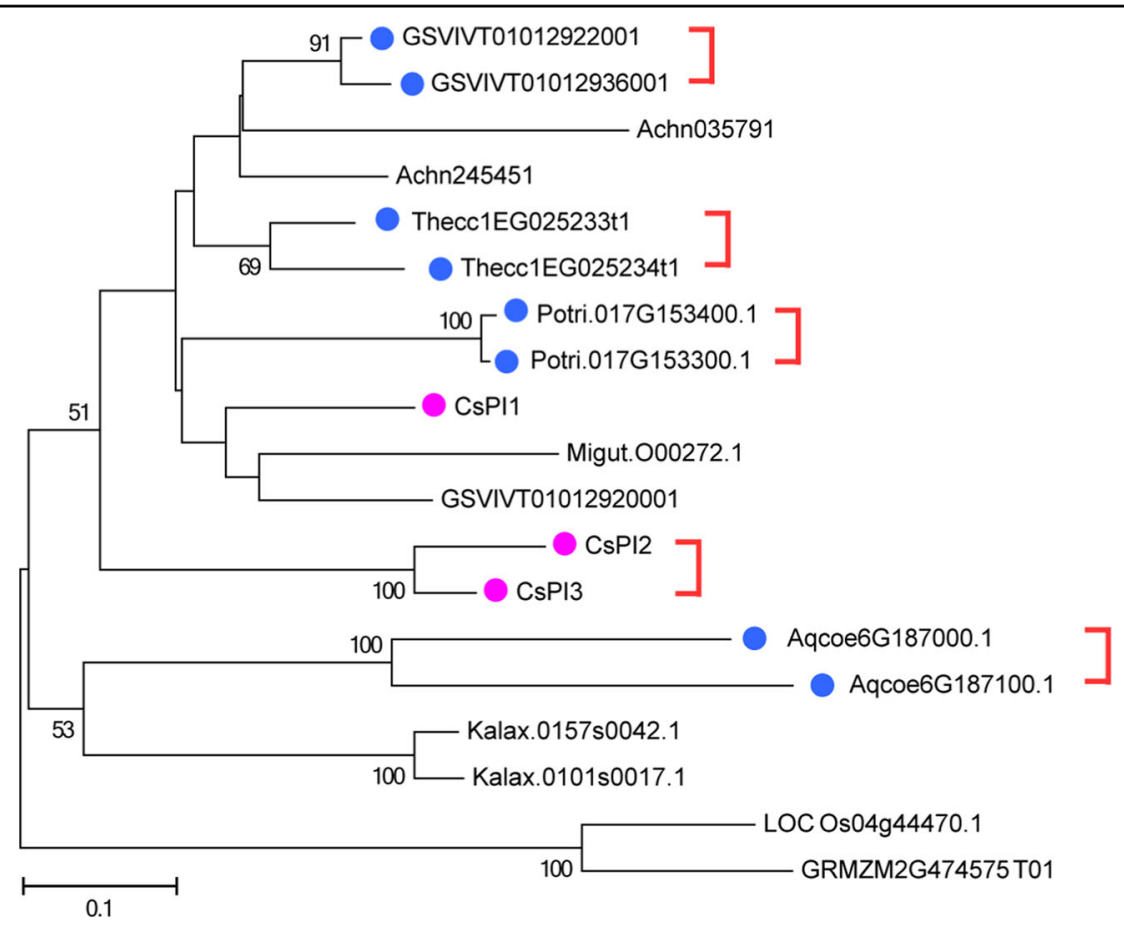

Fig. 2 Phylogenetic tree of CsKPI genes in tea plant. Nineteen KPI proteins from ten plant species were used to construct an unrooted neighbor joining phylogenetic tree. The bootstrap values of the confidence levels are shown as percentages at branch nodes. The CsKPI genes are highlighted in purple. The red half-frame indicates a tandem duplication event between the two framed genes. The solid blue circles indicate tandem duplications of genes from other plant species

Sodium dodecyl sulfate polyacrylamide gel electrophoresis analysis indicated that the expressed proteins were of the expected sizes (including the GST tag): CsKPI1, $49 \mathrm{kDa}$; CsKPI2, $49 \mathrm{kDa}$; CsKPI3, $50 \mathrm{kDa}$ (Fig. 3a and Supplementary Fig. S2). To investigate the trypsin inhibitory activity of the CsKPI proteins, we performed highperformance liquid chromatography (HPLC) analysis to test the products resulting from mixed enzymatic reactions. Based on their identical reaction conditions, the enzymatic reactions with CsKPI proteins yielded a smaller HPLC peak than those of the blank control sample and the empty vector control sample pGEX-4T-1 (Fig. 3b). Quantitative determination of the PNA content based on peak area and standard curve analysis also supported these results (Fig. 3c), and we concluded that the enzymatic reaction was inhibited by these three CsKPI proteins. Further quantitative determination indicated that the specific inhibitory activities of CsKPI1, CsKPI2, and CsKPI3 recombinant proteins were 499.87, 957.62, and 1694.78 units/g, respectively. To assess the function of the two conserved residues (Arg and Lys), these residues were mutated by site-directed mutagenesis for three CsKPI constructs, and the recombinant proteins were expressed in E. coli (Supplementary Fig. S3A). Further results of the enzymatic assay showed that the inhibitory activities of the CsKPI2 and CsKPI3 mutant proteins were significantly decreased relative to those of the respective wild-type proteins; this decrease was not obviously observed in the CsKPI1 mutant protein (Supplementary Fig. S3B, C). To further investigate the subcellular localization of the three CsKPI proteins, we constructed CsKPI1-GFP, CsKPI2-GFP, CsKPI3-GFP, and pK7WGF2 $35 S-G F P$ fusion protein expression vectors. Transient expression in Arabidopsis protoplasts showed that the CsKPI1-GFP, CsKPI2-GFP, and CsKPI3-GFP proteins localized throughout the cytoplasm without distinction (Fig. 3d). Prediction of the signal peptide using SignalP and TargetP software suggested that they may be involved in the secretory pathway (Supplementary Table. S1).

\section{CSKPI2 and CSKPI3 are involved in defense responses against pathogen infection by $S A$ induction}

We explored the expression patterns of CsKPI genes in response to Glomerella cingulata (fungal causative agent of brown blight disease) infection using qRT-PCR analysis. The three CsKPIs were differentially expressed under this treatment. CsKPI2 and CsKPI3 were strongly induced at 1 day post inoculation; in particular, the mRNA of CsKPI2 in infected leaves accumulated up to 19.5-fold relative to that in the untreated control leaves (Fig. 4). Specifically, the expression of CsKPI2 and CsKPI3 reached peaks of up to 26.5-fold and 7.6-fold higher than those of 


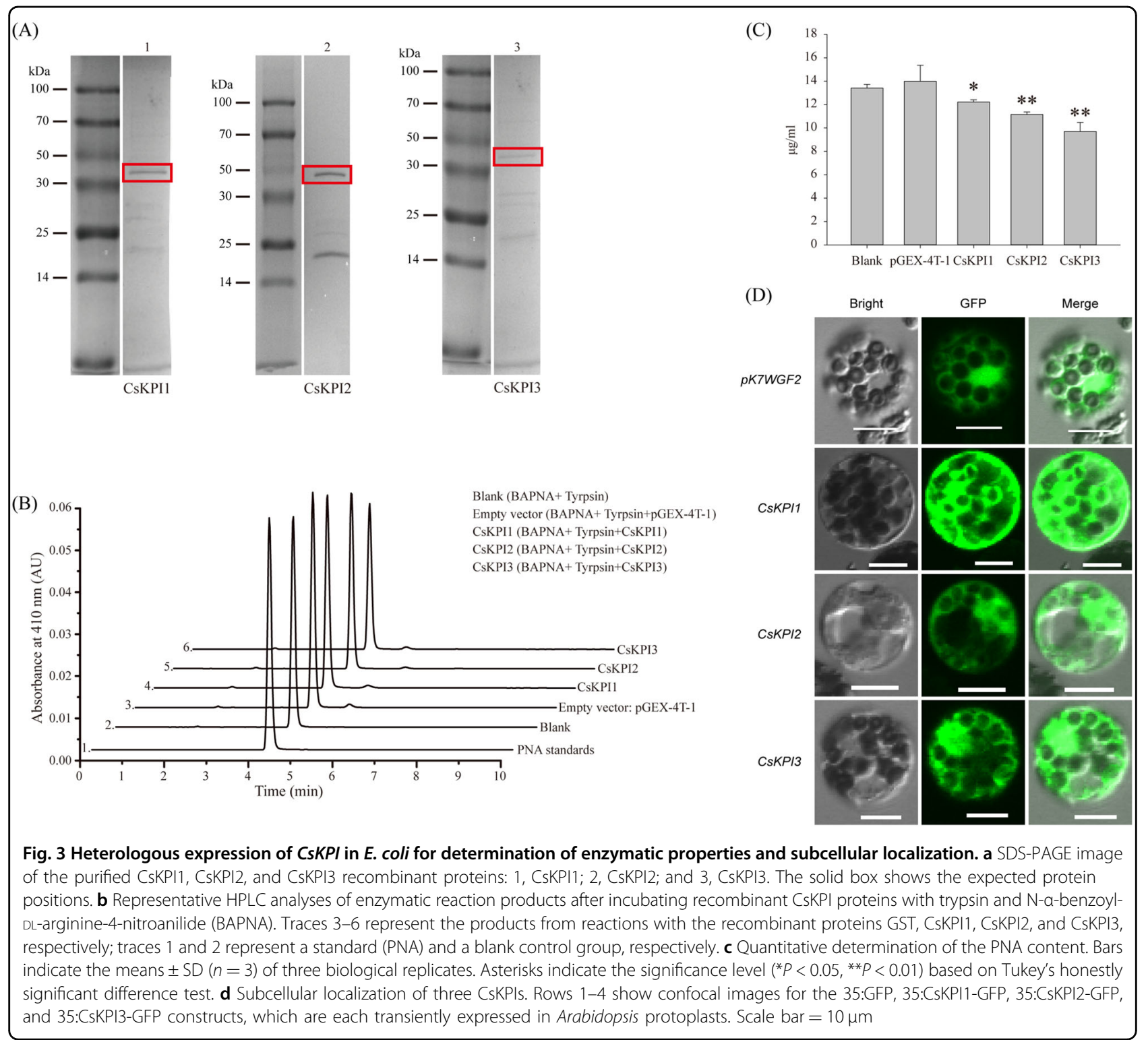

the control at 10 days post inoculation, respectively; however, CsKPI1 was only 1.1-fold expression at 10 days post inoculation (Fig. 4), suggesting the functional diversity among them in response to pathogen infection.

The SA content in infected leaves increased compared to that in healthy leaves (as a control) (Supplementary Fig. S4A), while the ABA, JA, and JA-Ile contents showed nonsignificant increases, respectively (Supplementary Fig. S4B-D). To investigate the influence of SA accumulation on the expression of CsKPIs, qRT-PCR was performed. CsKPI2 and CsKPI3 were strongly induced at $12 \mathrm{~h}$ after SA treatment (Supplementary Fig. S5A); moreover, TCA motifs (SA responsiveness element) were found in the promoter sequences of both of them. To further confirm the induction of CsKPI2 and CsKPI3 by SA, the promoters of CsKPI2 and CsKPI3 were cloned based on their genomic sequences and placed upstream of a GFP reporter gene and were transiently expressed in Arabidopsis protoplast cells with and without SA treatment. The results showed that $C s K P I 2_{p^{-}}$and $C s K P I 3_{p}$-driven GFP were strongly expressed in SA-treated protoplast cells but not in untreated cells. In addition, the protoplasts expressing CaMV35S : :GFP showed ubiquitous expression in cells irrespective of SA treatment (Fig. 5a).

\section{JA induced the expression of the CsKPI1 gene during tea geometrid feeding}

Since CsKPI genes were shown to be involved in insect feeding defense reactions ${ }^{38}$, we used starved tea geometrids to damage healthy leaves. The results of qRT-PCR 


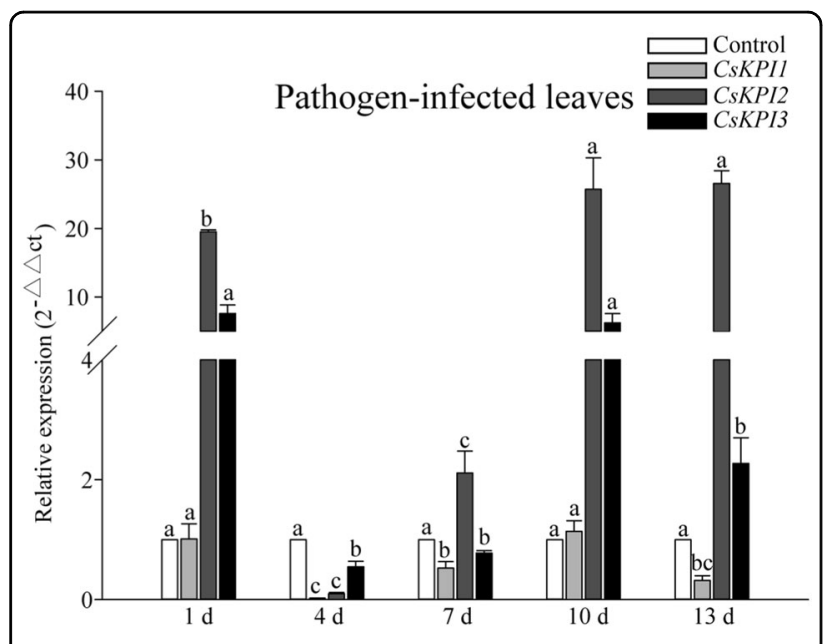

Fig. 4 Expression patterns of CsKPI genes in leaves infected with Glomerella cingulata at different time points. QRT-PCR analysis of CSKPI mRNA transcripts in leaves subjected to Glomerella cingulata infection. Bars indicate the means \pm SD $(n=3)$ of three biological replicates, and bars with different letters are significantly different at $P<0.05$ according to Duncan's multiple range test showed induction of all three CsKPIs, but the expression levels of CsKPI1 quickly $(12 \mathrm{~h})$ reached a maximum in localized insect-fed leaves (Fig. 6a). To gain additional insight into the potential signal transduction function of the CsKPI genes during insect attack, we also performed qRT-PCR with undamaged neighboring leaves. Notably, CsKPI1 was not only induced in local insect-fed leaves, but the expression level was substantially higher in undamaged neighboring leaves. For example, the CsKPI1 mRNA level in insect-fed leaves was up to 1.9-fold that of the control, but a 70.8-fold upregulation was observed in the undamaged neighboring leaves (Fig. 6b). These results suggest that the CsKPI1 gene may play a role in signal transduction in the tea plant response to tea geometrid attack.

The accumulation of hormones is vital to signal transduction during plant attack by insects; hence, the contents of phytohormones were measured to investigate whether any of them could induce the expression of CsKPII in tea plant attacked by tea geometrid. We found that the JA and JA-Ile contents were significantly increased in both
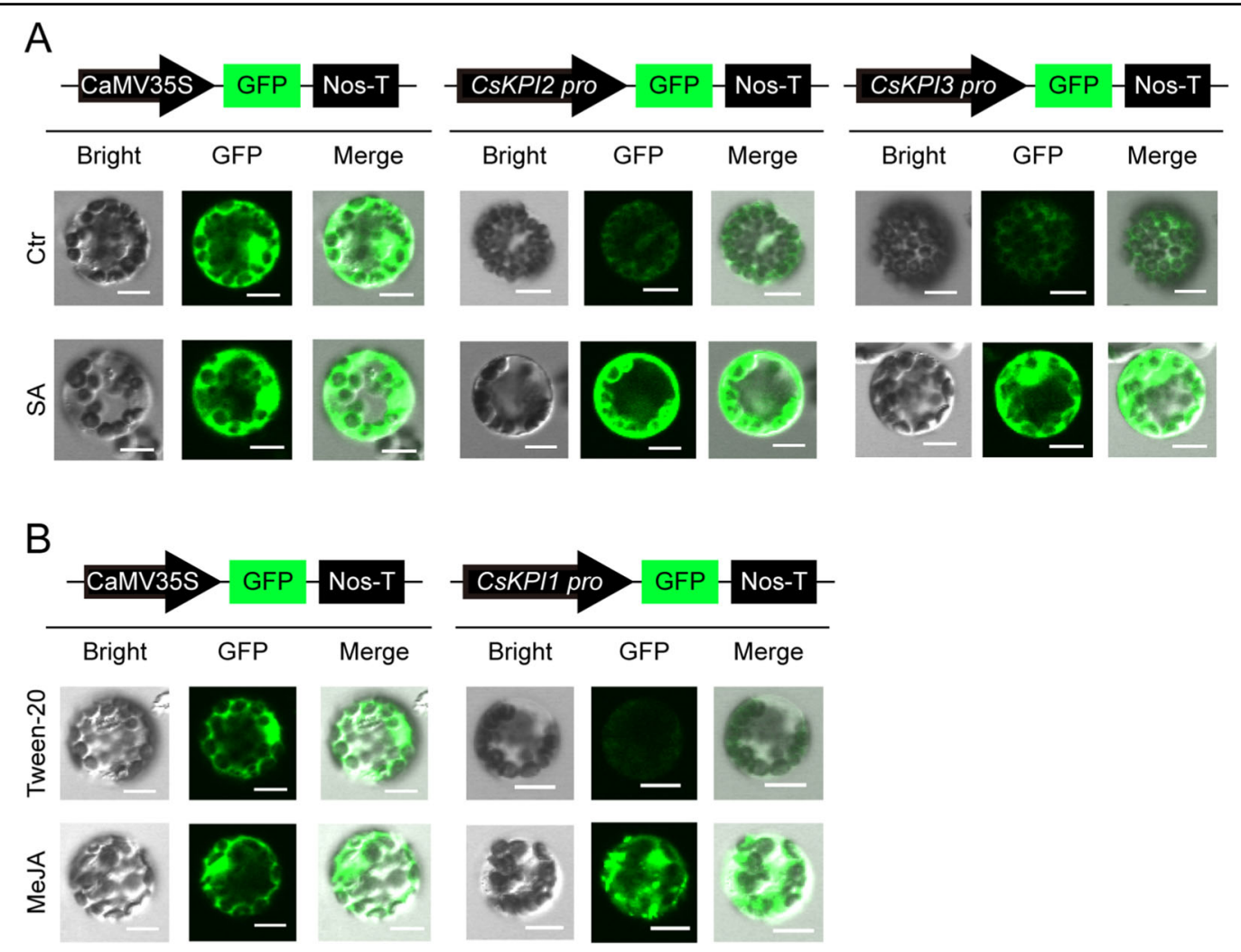

Fig. 5 Detection of CSKPI promoter activities transiently expressed in Arabidopsis protoplasts. GFP reporter constructs containing CSKPII, CSKPI2, and CSKPI3 promoters (CSKPI1 pro-GFP, CSKPI2 pro-GFP, and CSKPI3 pro-GFP) and the CaMV35S promoter (35S pro-GFP; positive control) were used to transiently transfect Arabidopsis protoplasts, which were tested for SA (a) and MeJA (b) induction. After incubation for $8 \mathrm{~h}$, the GFP signal was observed by confocal microscopy. Ctr, control group with sterilized water treatment. Bars $=10 \mu \mathrm{m}$ 

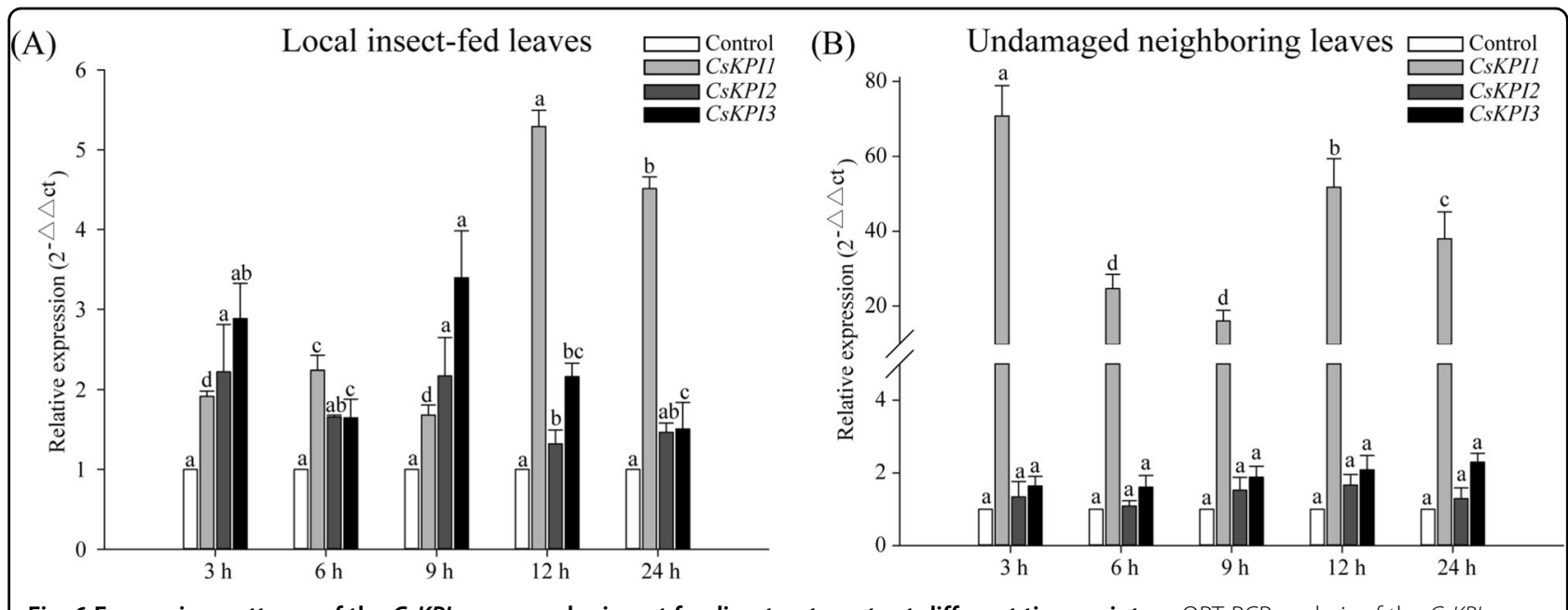

Fig. 6 Expression patterns of the CsKPI genes under insect feeding treatments at different time points. a QRT-PCR analysis of the CsKPI genes in damaged leaves under insect feeding treatment. $\mathbf{b}$ QRT-PCR analysis of the CSKPI genes under insect feeding treatment in undamaged neighboring leaves. Bars indicate the means $\pm \mathrm{SD}(n=3)$ of three biological replicates. Different letters above the bars denote significant differences at $P<0.05$ according to Duncan's multiple range test

localized damaged leaves and neighboring undamaged leaves (Fig. 7), but no induction of ABA and SA was observed (Supplementary Fig. S6A-D). Although high contents of JA and JA-Ile were detected in undamaged neighboring leaves, their amounts were higher in localized insect-fed leaves, suggesting an attenuation of the defense signal with time and distance from the source under insect attack. Moreover, CsKPI1 was upregulated under MeJA treatment in both treated and untreated neighboring leaves, but no induction was observed for CsKPI2 and CsKPI3 (Supplementary Fig. S5B, C). This implies that JA acts as a CsKPI1-specific signal molecule to modulate the expression of CsKPI1 and function in SAR defense. In support of these data, MeJA-responsive elements (CGTCA and TGACG motifs) were observed in the CsKPI1 promoter (Supplementary Table. S2). To determine whether the CsKPI1 promoter responded to MeJA signals, the CaMV35S promoter was substituted by the CsKPI1 promoter, and a transient GFP assay was conducted by introducing the chimeric gene into Arabidopsis protoplast cells. The GFP signal intensity of the CsKPI1 promoter in the transfected cells was significantly enhanced at $8 \mathrm{~h}$ after treatment, while the signal driven by the CaMV35S promoter was not significantly changed, indicating that CsKPI1 responded to MeJA signals (Fig. 5b).

\section{Discussion}

PIs have been characterized in many plant species and play key regulatory roles in plant development and in abiotic and biotic stresses ${ }^{11,33,39}$. KPIs form a separate subfamily and have been shown to perform vital defensive functions against pests ${ }^{8}$. However, little is known about the transcriptional differentiation of KPIs in response to insect attack and pathogen infection in plants. In this study, we identified and cloned three full-length CsKPI genes from the tea plant (C. sinensis) genome. The inhibitory efficiencies and subcellular localizations of these CsKPI proteins were experimentally verified. We noted differential expression patterns between the three CsKPIs in response to tea geometrid feeding and pathogen infection. We concluded that CsKPI1 was induced during tea leaf feeding by tea geometrids by determining the contents of three hormones in localized insect-damaged and neighboring undamaged leaves and by promoter activity analysis. Moreover, these results implied that pathogen infection activated the expression of CsKPI2 and CsKPI3. Thus, this study provides important details of the divergence of CsKPIs in tea plant and represents an example of the evolutionary employment of duplicated genes that seem to have similar physiological functions.

From the absence of KPIs in D. salina to the presence of three KPIs in C. sinensis, KPI genes have appeared and expanded into a family with the evolution of plants (Fig. 2). Two rounds of WGD occurred in the $C$. sinensis lineage. In contrast, $V$. vinifera, as a species with a close relationship with $C$. sinensis, did not experience a WGD event $^{3}$, and three similar KPI genes were found within its genome (Fig. 2). Therefore, the unexpected expansion of the KPI gene family in tea plant may not be caused by WGD events. Instead, the appearance of CsKPI2 and CsKPI3 were likely caused by a tandem duplication event that occurred 26.78 MYA. These genes have high sequence similarity, share the same location in the genome and have the same expression pattern in response to biotic stresses (Figs. 2, 4, and 5). Similarly, this tandem duplication event was conserved among other 


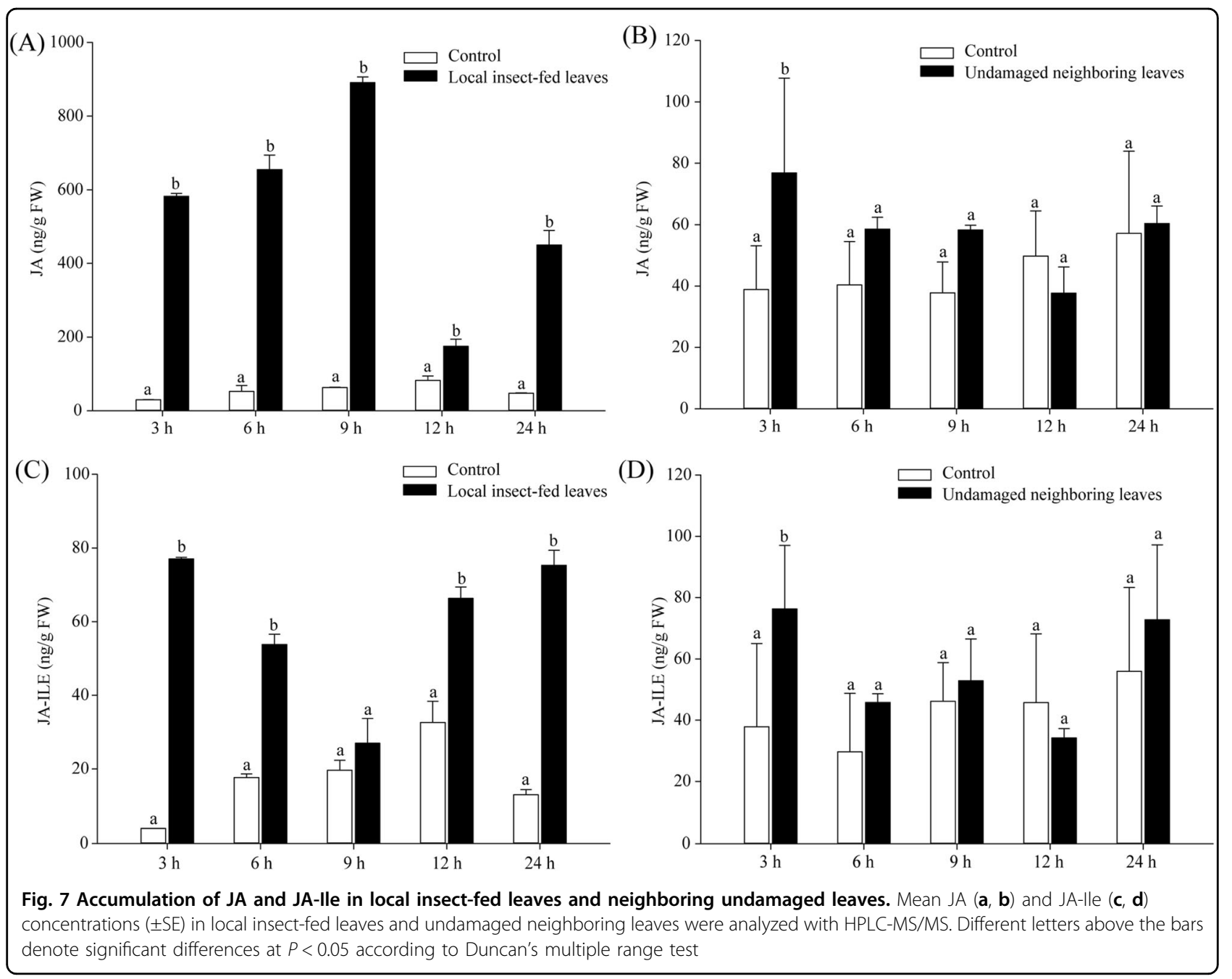

closely related plant species, such as $P$. trichocarpa (Potri.017G153400.1 and Potri.017G153300.1), V. vinifera (GSVIVT01012922001 and GSVIVT01012936001), and T. cacao (Thecc1EG025233t1 and Thecc1EG025234t1) (Fig. 2). The three CsKPI proteins suppress the activity of trypsin and reduce the formation rate of the PNA reaction product (Fig. 3b, c), which mirrors the activities of KPIs from other plant species ${ }^{40,41}$. In addition, the inhibitory activities of the CsKPI2 and CsKPI3 mutant proteins were nearly abolished after two key residues (Arg and Lys) were replaced with alanine, suggesting that these two residues have essential roles in the inhibition of PIs (Supplementary Fig. S3). Notably, these decreases in inhibitory activity were not found in the CsKPI1 protein after replacement of the homologous residues. These large differences in protein properties may result from their low amino acid sequence similarity and the distant evolutionary relationship between CsKPI1 and other CsKPIs. These results also implied that there were undefined key residues in the CsKPI1 protein that influenced its inhibitory activity.
Previous studies have indicated that some PI proteins are localized to the plasma membrane, cytoplasm or extracellular space ${ }^{42-45}$. Our transient expression data in Arabidopsis protoplast cells showed that all three CsKPI proteins were diffusely distributed in the cytoplasm, nucleus and plasma membrane, rather than localized to one organelle. This may have resulted from the presence of a signal peptide (Fig. 3d and Supplementary Table S1).

In many transgenic plants, PIs have been overexpressed to impart resistance against different pathogen infections $^{46,47}$. In this study, CsKPI2 and CsKPI3 were strongly induced in response to G. cingulata infection (Fig. 4), but no significant transcriptional changes were detected for CsKPI1. The results imply that natural selective pressure might result in the transcriptional differentiation between CsKPI1 and the other two CsKPIs, which is similar to the previous observations in the phenylalanine ammonia-lyase and expansin gene families ${ }^{26,27}$. Based on cis-element analysis of CsKPI2 and CsKPI3, the presence of the fungal elicitor element (Box-W1) in the promoter could be a 
factor for sensitivity to pathogen infection (Supplementary Table S2) ${ }^{48}$. Furthermore, we found that out of the four hormones, only SA accumulated in infected leaves 13 days after inoculation, and SA induced the expression of CsKPI2 and CsKPI3 at the transcriptional level (Supplementary Fig. 5A and Fig. 5a). Since SA is a well-known phytohormone regulating disease resistance in plants ${ }^{29}$, our results suggested that SA may be the main hormone signal mediating the expression of CsKPI2 and CsKPI3 to defend against pathogen infection. The tandem duplication leading to the CsKPI2 and CsKPI3 genes may confer enhanced resistance in tea plant against various pathogens.

In plants, PIs have been commonly considered as antiinsect proteins, which interfere with the digestive processes of insects ${ }^{38}$. Similarly, the expression of the three CsKPIs was significantly upregulated during tea geometrid feeding (Fig. 6a), but the highest transcription level was observed for CsKPI1. These results further support our hypothesis that transcriptional differentiation occurred after the divergence of the three CsKPIs from their common ancestor 72.94 MYA. The recent tandemly duplicated CsKPI2 and CsKPI3 genes are primarily involved in defense against pathogen infection, and CsKPI1 is associated with resistance against insect attack. Furthermore, we simultaneously found that CsKPI1 was more intensely induced in undamaged neighboring leaves (Fig. 6b). These results suggest that the CsKPI1 gene not only contributes to the defense response in localized insect-fed leaves but is also involved in SAR during tea geometrid attack.

Studies have shown a linkage between JA accumulation and the expression of PIs in response to insect $\operatorname{attack}^{33,49,50}$. Moreover, high abundances of JA and CsKPI1 were simultaneously detected in localized insectdamaged leaves. The contents of JA and JA-Ile both increased in undamaged neighboring leaves (Fig. 7), while no significant accumulation of ABA and SA was detected (Supplementary Fig. S6A-D), suggesting that JA is the vital hormone signal that imparts resistance to herbivory in localized insect-damaged tissue and in the SAR defense response and that JA induces the expression of CsKPI1 in undamaged neighboring leaves. JA transport may occur within the bulk flow of the phloem or in the transpiration stream, thereby facilitating systemic signal transmis$\operatorname{sion}^{51,52}$. In support of our hypothesis, the JA derivative MeJA could induce the expression of the CsKPI1 promoter and initiate the expression of a downstream GFP reporter gene (Supplementary Fig. 4B). JA would then mediate a signaling cascade to activate the expression of CsKPI1 and result in SAR to initialize the various systemwide defense responses in tea plant.

In conclusion, we identified three CsKPIs in tea plant, investigated their evolutionary relationship, and characterized these CsKPIs by heterologous expression. CsKPI2 and CsKPI3 were generated through tandem duplication and show similar expression patterns that facilitate the tea plant defense response to pathogen infection. In contrast, CsKPI1 transcriptionally differs from CsKPI2 and CsKPI3 and participates in the SAR response, which requires JA accumulation resulting from tea geometrid feeding (Fig. 8).

\section{Materials and methods \\ Plant materials and treatments}

Two-year-old tea plants (Camellia sinensis cv. Shuchazao) were obtained from the Dechang tea plantation (Shucheng, latitude $31.3^{\circ} \mathrm{N}$, longitude $117.2^{\circ} \mathrm{E}$, Anhui, China). Tea plants were grown outdoors in an experimental plot with $120 \mathrm{~cm}$ between rows and $33 \mathrm{~cm}$ between plants within a row. All tea plants were watered and fertilized with the same routine. The plants chosen for our experiments had uniform heights and canopy widths and were without signs of disease and insects.

Tea geometrids were captured in fields at the Dechang tea plantation and were identified as Ectropis oblique. A population of tea geometrids was reared in a growth chamber (temperature: $25^{\circ} \mathrm{C}$, photoperiod: $14 / 10 \mathrm{~h}$ (day/ night), humidity: $65 \pm 5 \%$ ). For the insect feeding treatment, 20 tea geometrids at the third larval stage were placed on selected leaves of each individual tea plant. After $1 / 3$ of each leaf was consumed, all tea geometrids were removed, and then the consumed leaves and the undamaged neighboring leaves below them were collected at $3,6,9,12$, and $24 \mathrm{~h}$. The samples in the control group without tea geometrid feeding were collected at the same time points (Supplementary Fig. S7A).

The G. cingulata fungal strain was obtained from our laboratory's strain store room. The tea plants were infected according to a previously published protocol developed by our team ${ }^{53}$. Plants were incubated in the greenhouse (temperature: $28 \pm 3^{\circ} \mathrm{C}$, photoperiod: $16 / 8 \mathrm{~h}$ (day/night), humidity: $85 \pm 5 \%$ ). The control group consisted of plants with healthy leaves that were inoculated with sterile water. Infected and uninfected control leaves were collected on days 1, 4, 7, 10, and 13 days (Supplementary Fig. S7B).

Leaves of healthy tea plants were wiped with $1 \mathrm{mM}$ MeJA (in $0.05 \%$ Tween-20) or $1 \mathrm{mM} \mathrm{SA}$ until the leaves were completely wet. Leaves treated with $0.05 \%$ Tween- 20 and double-distilled water served as the controls for both treatments. The treated leaves and the untreated neighboring leaves below them were collected at 3, 6, 9, 12, and $24 \mathrm{~h}$ (Supplementary Fig. S7C).

Three biological replicates were performed for all treatments, and all collected samples were immediately frozen in liquid nitrogen and stored at $-80^{\circ} \mathrm{C}$ until use.

\section{Bioinformatics analysis}

To investigate the evolutionary divergences among the three CsKPIs, synonymous substitution rates (Ks) of three 

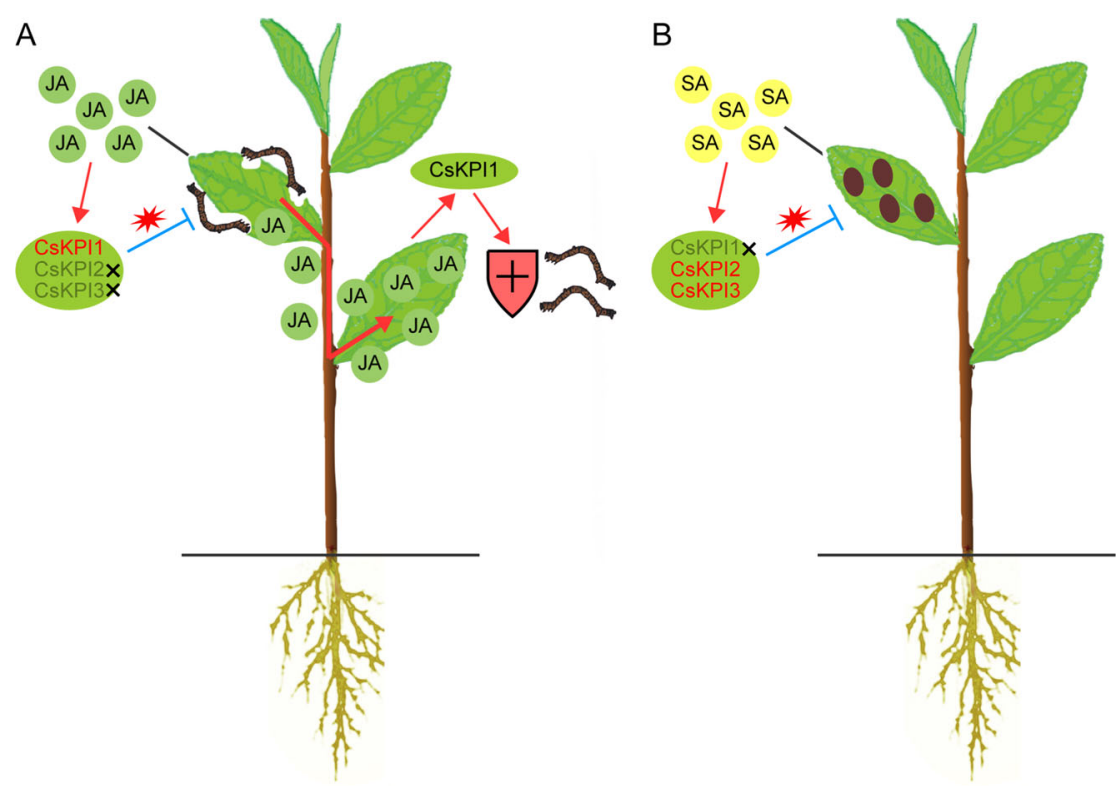

Fig. 8 A hypothetical model for function of CSKPI genes in tea plant. a Tea geometrid feeding stimulates the accumulation of JA, which subsequently induces the expression of CSKPII to defend against tea geometrid attack by repressing their digestive systems. The JA produced in local insect-fed leaves would be transported into undamaged neighboring leaves or biosynthesized de novo to induce CsKPII expression and trigger a broader defense response against tea geometrids. b Glomerella cingulate induces the accumulation of SA, which activates the transcription of CSKPI2 and CSKPI3 to defend the tea leaves from pathogen infection

CsKPIs were calculated using KaKs_Calculator 2.0 based on the MA model. The divergence time was converted based on the calculated Ks value according to $T=K s / 2 r$, where $r$ indicates a substitution rate of $6.5 \times 10^{-9}$ mutations per site per year for eudicots.

MEGA 6.0 software was employed to construct a phylogenetic tree after the multiple sequence alignment analyses of CsKPIs. In the phylogenetic tree, 19 KPI CDS protein sequences were analyzed as candidate genes using the neighbor joining method set to the following parameters: complete deletion, uniform rates, Poisson model, bootstrap method (1000 replicates). The gene accession information for all genes is listed in Supplementary Table S3.

\section{Total RNA extraction and qRT-PCR analysis}

The RNAprep Pure Plant Kit (cat DP441, Tiangen) was used to extract total RNA from plant materials according to the manufacturer's instructions. The PrimeScript RT Reagent Kit (cat 6110A, Takara) was used to synthesize first-strand cDNA from total RNA. QRT-PCR was performed with a protocol previously reported by our team ${ }^{6}$. All gene-specific primers (P1-P8) are listed in Supplementary Table S4. The amplification efficiencies of all genes used in this study ranged from 95 to $105 \%{ }^{54}$. DPS software was used to perform the statistical analysis of qRT-PCR data ${ }^{55}$.

\section{Gene cloning of full-length CsKPI genes and site-directed mutagenesis}

Putative CsKPIs were obtained based on the current assembly of $C$. sinensis genome sequences ${ }^{3}$. The fulllength sequence (P9-P10) was obtained using a SMART RACE Kit (cat 6107, Clontech, Dalian, China) (Supplementary Table S4). Gene-specific primers (P11-P16) were used to amplify full-length CsKPI gene sequences (Supplementary Table S4), and the CsKPI genes were separately cloned into the pEASY-T1 vector and verified by DNA sequencing.

Site-directed mutagenesis was performed on the CsKPI vectors to yield CsKPI1 (R98A, K152A), CsKPI2 (R99A, K155A), and CsKPI3 (R104A, K160A). In brief, three pairs of site-mutant primers were designed and used to amplify three DNA fragments, and then targeted CsKPI fragments were obtained by fusing the three DNA fragments using end-to-end PCR. The PCR products containing mutations were separately cloned into the pEASY-T1 vector and verified by DNA sequencing; the mutagenesis primers are listed in Supplementary Table S4.

\section{Heterologous expression and purification of the CsKPI proteins}

FastPfu polymerase (cat AP221, Transgen, Beijing, China) was used to amplify the ORFs of CsKPI1, CsKPI2, CsKPI3, and the three corresponding mutated CsKPIs 
using gene-specific primers (P17-P22) (Supplementary Table S4). The pGEX-4T-1 vector was selected as the expression vector; the six target genes (wild types and CsKPI mutants) and the vector were digested with the restriction enzymes BamHI (1010A, Takara) and XhoI (1094A, Takara). The ClonExpressII One Step Cloning Kit (cat C112, Vazyme) was used for the assembly according to the manufacturer's instructions. The constructed plasmids were transformed into E. coli BL21 chemically competent cells (cat CD601, Transgen). GST resin (cat DP201, Transgen) was used to purify the GSTtagged recombinant proteins by affinity chromatography.

\section{Enzyme activity assay}

The substrate BAPNA (B3133, Sigma Aldrich) was dissolved in dimethylsulfoxide (DMSO) and added to $50 \mathrm{mM}$ Tris-HCL, $25 \mathrm{mM} \mathrm{CaCl}_{2}, \mathrm{pH} 8.0$, to a final concentration of $0.4 \mathrm{mg} / \mathrm{mL}$. BAPNA $(200 \mu \mathrm{L}, 0.4 \mathrm{mg} / \mathrm{mL})$ and the purified protein solution $(100 \mu \mathrm{L}, 0.4 \mu \mathrm{g} / \mu \mathrm{L})$ were mixed and incubated at $37^{\circ} \mathrm{C}$ for $10 \mathrm{~min}$. Trypsin $(200 \mu \mathrm{L}, 250 \mathrm{U} / \mu \mathrm{L})$ was then added, and the mixture was incubated at $37^{\circ} \mathrm{C}$ for another $30 \mathrm{~min}$. Finally, 30\% glacial acetic acid $(100 \mu \mathrm{L})$ was added to stop the enzymatic reaction. The catalytic reaction product PNA was measured by HPLC, which was performed on a Waters series HPLC system with a C18 column and ultraviolet detection set at $410 \mathrm{~nm}$. The mobile phase consisted of $1 \%$ acetic acid aqueous solutionacetonitrile $(50: 50 \mathrm{v} / \mathrm{v})$ at a flow rate of $1 \mathrm{~mL} / \mathrm{min}^{40,41}$. The column oven temperature was set at $25^{\circ} \mathrm{C}$.

\section{Subcellular localization of the GFP fusion proteins}

Gene-specific primers, P23-P28 (Supplementary Table S4), were used to amplify ORFs of CsKPI1, CsKPI2, and CsKPI3, and the PCR products were separately inserted into the pK7WGF2 vector by the BP and LR reactions using Gateway Technology. The resultant plasmids pK7WGF2-KPI1, pK7WGF2-KPI2, and pK7WGF2-KPI3 were transformed into Arabidopsis protoplast cells according to a previously reported protocol ${ }^{56}$. After overnight transformation, protoplasts were examined using an Olympus FV1000 confocal microscope (Olympus, Japan).

\section{Analysis of phytohormone concentrations}

The JA, JA-Ile, ABA, and SA concentrations were measured in three biological replicates according to reported studies ${ }^{57}$. Approximately, $150 \mathrm{mg}$ of frozen leaf material was ground in liquid nitrogen; ethyl acetate $(1 \mathrm{~mL})$ containing $\mathrm{D}_{5}$-JA, ${ }^{13} \mathrm{C}_{6}$-JA-Ile, $\mathrm{D}_{6}$ - $\mathrm{ABA}$, and $\mathrm{D}_{4}$ SA was added to each sample to serve as internal standards. Samples were mixed and then centrifuged at $13,000 \mathrm{~g}$ for $10 \mathrm{~min}$ at $4{ }^{\circ} \mathrm{C}$. The supernatants were evaporated to dryness in a vacuum concentrator (Eppendorf, Germany). The residues from the samples were resuspended in $0.4 \mathrm{~mL}$ of $70 \%$ methanol $(\mathrm{v} / \mathrm{v})$, and the samples were centrifuged again to clarify the phases. Phytohormone concentrations were measured on an LCMS8040 (Shimadzu, Japan) with a Shim-pack XR-ODS column $(2.0 \times 75 \mathrm{~mm}, 2.2 \mu \mathrm{m})$ (Shimadzu, Japan). The column temperature was set at $40{ }^{\circ} \mathrm{C}$, and the flow rate was $0.27 \mathrm{~mL} / \mathrm{min}$.

\section{Promoter activity assays}

The CP Plant Miniprep Kit (cat GD2621-01, BIOMIGA) was used to extract genomic DNA from tea leaves according to the manufacturer's instructions. The promoters of CsKPI1, CsKPI2, and CsKPI3 were amplified from genomic DNA with FastPfu polymerase using promoter-specific primers (P29-P34) (Supplementary Table S4). The CsKPI1, CsKPI2, and CsKPI3 promoter amplicons were separately inserted into the $p C A M B I A 1302-G F P$ vector by replacing the CaMV35S promoter, and they were then used to transform Arabidopsis protoplasts as previously described ${ }^{58}$. Transformed protoplasts were treated with $0.05 \%$ Tween-20 (control), $50 \mu \mathrm{M}$ MeJA and $50 \mu \mathrm{M} \mathrm{SA}$ for $8 \mathrm{~h}$ at $25^{\circ} \mathrm{C}$. The GFP signal was detected after phytohormone treatment using an Olympus FV1000 confocal microscope.

\section{Acknowledgements}

This work was supported by the National Key Research and Development Program of China (2018YFD1000601), the National Natural Science Foundation of China (31171608), the Special Innovative Province Construction in Anhul Province (15czs08032), the Special Project for Central Guiding Science and Technology Innovation of Region in Anhui Province (2016080503B024), and the Program for Changjiang Scholars and Innovative Research Team in University (IRT1101).

\section{Conflict of interest}

The authors declare that they have no conflict of interest.

Supplementary Information accompanies this paper at (https://doi.org/ 10.1038/s41438-019-0208-5).

Received: 2 February 2019 Revised: 10 September 2019 Accepted: 18 September 2019

Published online: 15 November 2019

\footnotetext{
References

1. Shi, C. Y. et al. Deep sequencing of the Camellia sinensis transcriptome revealed candidate genes for major metabolic pathways of tea-specific compounds. BMC Genomics 12, 131 (2011).

2. Wheeler, D. S. \& Wheeler, W. J. The medicinal chemistry of tea. Drug Dev. Res. 61, 45-65 (2004).

3. Wei, C. et al. Draft genome sequence of Camellia sinensis var. sinensis provides insights into the evolution of the tea genome and tea quality. Proc. Natl Acad. Sci. USA 115, E4151-E4158 (2018).

4. Liu, S. C. et al. Transcriptomic analysis of tea plant responding to drought stress and recovery. PLOS ONE 11, e0147306 (2016).

5. Ban, Q. et al. Comparative analysis of the response and gene regulation in cold resistant and susceptible tea plants. PLOS ONE 12, e0188514 (2017).

6. Wang, Y. N. et al. Differential transcriptome analysis of leaves of tea plant (Camellia sinensis) provides comprehensive insights into the defense responses to Ectropis oblique attack using RNA-Seq. Funct. Integr. Genomics 16, 383-398 (2016b).
} 
7. Wang, L. et al. Transcriptome analysis of an Anthracnose-resistant tea plant cultivar reveals genes associated with resistance to Colletotrichum camelliae. PLOS ONE 11, e0148535 (2016a).

8. Habib, H. \& Majid, K. Plant protease inhibitors: a defense strategy in plants. Biotechnol. Mol. Biol. Rev. 2, 68-85 (2007).

9. Rustgi, S. et al. The complex world of plant protease inhibitors: Insights into a Kunitz-type cysteine protease inhibitor of Arabidopsis thaliana. Communicative Integr. Biol. 11, e1368599 (2017).

10. Grosse-Holz, F. M. \& van der Hoorn, R. A. Juggling jobs: roles and mechanisms of multifunctional protease inhibitors in plants. New Phytologist 210, 794-807 (2016).

11. Joshi, B. N. et al. Cysteine protease inhibitor from pearl millet: a new class of antifungal protein. Biochem. Biophys. Res. Commun. 246, 382-387 (1998).

12. Seldal, T. et al. Wound-induced proteinase inhibitors in Grey Alder (Alnus incana): a defence mechanism against attacking insects. Oikos 71, 239-245 (1994).

13. Vercammen, D. et al. Serpin1 of Arabidopsis thaliana is a suicide inhibitor for metacaspase 9. J. Mol. Biol. 364, 625-636 (2006).

14. Zhang, Y. et al. Effects of extrusion and expelling on the nutritional quality of conventional and Kunitz trypsin inhibitor-free soybeans. Poult. Sci. 72, 2299-2308 (1993).

15. Oliveira, A. S. et al. Two Kunitz-type inhibitors with activity against trypsin and papain from Pithecellobium dumosum seeds: purification, characterization, and activity towards pest insect digestive enzyme. Protein Pept. Lett. 16, 1526-1532 (2009).

16. Hermosa, M. R. et al. Identification and characterization of potato protease inhibitors able to inhibit pathogenicity and growth of Botrytis cinerea. Physiol. Mol. Plant Pathol. 68, 138-148 (2006).

17. Blanc, G. \& Wolfe, K. H. Functional divergence of duplicated genes formed by polyploidy during Arabidopsis evolution. Plant Cell 16, 1679-1691 (2004)

18. Lynch, M. \& Conery, J. S. The evolutionary fate and consequences of duplicate genes. Science 290, 1151-1155 (2000).

19. Taylor, J. S. \& Raes, J. Duplication and divergence: the evolution of new genes and old ideas. Annu. Rev. Genet. 38, 615-643 (2004).

20. Xiaohan, Y. et al. Divergence of the Dof gene families in poplar, Arabidopsis, and rice suggests multiple modes of gene evolution after duplication. Plant Physiol. 142, 820-830 (2006).

21. Hughes, A. L. The Evolution of functionally novel proteins after gene duplication. Proc. Biol. Sci. 256, 119-124 (1994).

22. Force, A. et al. Preservation of duplicate Genes by complementary, degenerative mutations. Genetics 151, 1531-1545 (1999).

23. Haberer, G. et al. Transcriptional similarities, dissimilarities, and conservation of cis-elements in duplicated genes of Arabidopsis. Plant Physiol. 136, 3009-3022 (2004).

24. Madlung, A. et al. Genomic changes in synthetic Arabidopsis polyploids. Plant J. 41, 221-230 (2005).

25. Olga, P. et al. Chromosomal locus rearrangements are a rapid response to formation of the allotetraploid Arabidopsis suecica genome. Proc. Natl Acad. Sci. USA 101, 18240-18245 (2004).

26. Qing-Mao, S. et al. Multiple tandem duplication of the phenylalanine ammonia-lyase genes in Cucumis sativus L. Planta 236, 1093-1105 (2012).

27. Zhu, Y. et al. Soybean (Glycine max) expansin gene superfamily origins: segmental and tandem duplication events followed by divergent selection among subfamilies. BMC Plant Biol. 14, 1-19 (2014).

28. Shan, $X$. et al. Comparison of phytohormone signaling mechanisms. Curr. Opin. Plant Biol. 15, 84-91 (2012).

29. Vlot, A. C. et al. Salicylic Acid, a multifaceted hormone to combat disease. Annu. Rev. Phytopathol. 47, 177-206 (2009).

30. Goossens, J. et al. Jasmonates: signal transduction components and their roles in environmental stress responses. Plant Mol. Biol. 91, 673-689 (2016).

31. Pauwels, L. et al. Jasmonate-inducible gene: what does it mean? Trends Plant Sci. 14, 87-91 (2009).

32. Luo, J. et al. COl1-regulated hydroxylation of jasmonoyl-L-isoleucine impairs Nicotiana attenuata's resistance to the generalist herbivore Spodoptera litura. J. Agric. Food Chem. 64, 2822-2831 (2016).

33. Yang, D.-H. et al. BAK1 regulates the accumulation of jasmonic acid and the levels of trypsin proteinase inhibitors in Nicotiana attenuata's responses to herbivory. J. Exp. Bot. 62, 641-652 (2011).
34. Hilleary, R. \& Gilroy, S. Systemic signaling in response to wounding and pathogens. Curr. Opin. Plant Biol. 43, 57-62 (2018).

35. Fu, Z. Q. \& Dong, X. Systemic acquired resistance: turning local infection into global defense. Annu. Rev. Plant Biol. 64, 839-863 (2013).

36. Savatin, D. V. et al. Wounding in the plant tissue: the defense of a dangerous passage. Front. Plant Sci. 5, 470 (2014).

37. Truman, W. et al. Arabidopsis systemic immunity uses conserved defense signaling pathways and is mediated by jasmonates. Proc. Natl Acad. Sci. USA 104, 1075-1080 (2007).

38. Hines, M. E. et al. Isolation and partial characterization of a soybean cystatin cysteine proteinase inhibitor of coleopteran digestive proteolytic activity. J. Agric. Food Chem. 39, 1515-1520 (1991).

39. Boex-Fontvieille, E. et al. A Kunitz-type protease inhibitor regulates programmed cell death during flower development in Arabidopsis thaliana. J. Exp. Bot. 66, 6119-6135 (2015).

40. Lin, Z. Y. \& Yu, Y. Q. An HPLC method for the assay of trypsin inhibitors and its application to the study of Momordica cochinchinensis extract. Chin. J. Pharm. 20, 1151-1156 (2011)

41. Shamsi, T. N. et al. Allium sativum protease inhibitor: a novel Kunitz trypsin inhibitor from garlic is a new comrade of the Serpin family. PLOS ONE 11, e0165572 (2016).

42. Bhat, A. V. \& Pattabiraman, T. N. Protease inhibitors from jackfruit seed (Artocarpus integrifolia). J. Biosci. 14, 351-365 (1989).

43. Kim, N. H. \& Hwang, B. K. Pepper pathogenesis-related protein 4c is a plasma membrane-localized cysteine protease inhibitor that is required for plant cell death and defense signaling. Plant J. 81, 81-94 (2015).

44. Lampl, N. et al. Set-point control of RD21 protease activity by AtSerpin1 controls cell death in Arabidopsis. Plant J. 74, 498-510 (2013).

45. Pszenny, V. et al. Subcellular localization and post-secretory targeting of TgPI, a serine proteinase inhibitor from Toxoplasma gondii. Mol. Biochem. Parasitol. 121, 283-286 (2002).

46. Hilder, V. A. et al. A novel mechanism of insect resistance engineered into tobacco. Nature 330, 160-163 (1987).

47. Haq, S. K. et al. Protein proteinase inhibitor genes in combat against insects, pests, and pathogens: natural and engineered phytoprotection. Arch. Biochem. Biophys. 431, 145-159 (2004).

48. Kirsch, C. et al. A highly specific pathogen-responsive promoter element from the immediate-early activated CMPG1 gene in Petroselinum crispum. Plant J. 26, 217-227 (2010).

49. Ankala, A. et al. Foliar herbivory triggers local and long distance defense responses in maize. Plant Sci. 199-200, 103-112 (2013).

50. Heidel, A. J. \& Baldwin, I. T. Microarray analysis of salicylic acid- and jasmonic acid-signalling in responses of Nicotiana attenuata to attack by insects from multiple feeding guilds. Plant Cell Environ. 27, 1362-1373 (2004).

51. Koo, A. J. \& Howe, G. A. The wound hormone jasmonate. Phytochemistry 70 , 1571-1580 (2009).

52. Ferrieri, A. P. et al. Plant vascular architecture determines the pattern of herbivore-induced systemic responses in Arabidopsis thaliana. PLOS ONE 10 e0123899 (2015).

53. Zhu, J. et al. Characterization and alternative splicing profiles of lipoxygenase gene family in tea plant (Camellia sinensis). Plant Cell Physiol. 59, 1765-1781 (2018).

54. Kubista, M. et al. The real-time polymerase chain reaction. Mol. Asp. Med. 27, 95-125 (2006).

55. Tang, Q. Y. \& Zhang, C. X. Data processing system (DPS) software with experimental design, statistical analysis and data mining developed for use in entomological research. Insect Sci. 20, 254-260 (2013).

56. Shen, $\mathrm{H}$. et al. Functional characterization of the switchgrass (Panicum virgatum) R2R3-MYB transcription factor PVMYB4 for improvement of lignocellulosic feedstocks. New Phytologist 193, 121-136 (2012).

57. Wu, J. et al. Herbivory rapidly activates MAPK signaling in attacked and unattacked leaf regions but not between leaves of Nicotiana attenuata. Plant Cell 19, 1096-1122 (2007).

58. Yoo, S. D., Cho, Y. H. \& Sheen, J. Arabidopsis mesophyll protoplasts: a versatile cell system for transient gene expression analysis. Nat. Protoc. 2, 1565-1572 (2007). 